\title{
EDITORIAL
}

\section{Radiation exposure to cardiologists: how it could be reduced}

\section{E Vano}

Interventional cardiologists are at greater risk from radiation exposure as a result of the procedures they undertake than most other medical specialists. Thus, any measures that may help to reduce radiation exposure are most welcome

t is well known that occupational doses of radiation in interventional procedures guided by fluoroscopy are the highest doses registered among medical staff using $x$ rays. ${ }^{12}$ Interventional cardiologists represent the most important group of medical specialists involved in such practices.

Scatter radiation levels in the vicinity of the patient may be quite high under normal working conditions. ${ }^{1}$ If protection tools and good operational measures are not used, and if several complex procedures are undertaken per day, radiation lesions of the eyes may result after several years of work, particularly when the equipment used is not designed for interventional practices. ${ }^{1{ }^{3}}$

Interventional $x$ ray systems specifically designed for interventional cardiology should be a "sine qua non" condition for safety. Recently, the International Electrotechnical Commission has produced a new standard relating to the safety of $x$ ray systems to be used in interventional practice. $^{4}$

Several aspects of radiation safety in the practice of cardiology have been addressed by the American College of Cardiology (ACC) in a consensus document endorsed by the North American Society of Pacing and Electrophysiology, the Society for Cardiac Angiography and Interventions, and the American Society of Nuclear Cardiology. ${ }^{5}$

\section{TRAINING IN RADIOLOGICAL PROTECTION IS A KEY ISSUE}

The European Commission has addressed the importance of training in radiological protection (RP), publishing a guideline with specific recommendations for accreditation of training programmes for interventional procedures. ${ }^{6}$ This guideline suggests specific learning objectives and 20-30 hours of training for interventional cardiologists. Recently, a pilot course was held in Luxembourg at the National Institute of Cardiac Surgery and Interventional Cardiology to give accreditation in RP to the interventional cardiologists (senior specialists in that case). The attendants considered the course's duration of 16 hours (two full days) to be more than sufficient, and even too lengthy for some attendants. This was probably because of their current experience as interventionists.
New cardiology systems are sophisticated, and manufacturers have included many technical features to reduce radiation doses. However, a lack of RP knowledge and the detailed operation of these systems can sometimes prevent interventionalists from gaining their full advantage.

In 2001, an editorial in this journal addressed the radiation hazards for the patient in cardiological procedures, and stated that implementation of new training programmes in RP will be needed. ${ }^{7}$

The International Commission on Radiological Protection (ICRP) in its publication 85 states that "training in radiological protection for patients and staff should be an integral part of the education for those using interventional techniques" ... "a second, specific, level of training in radiation protection, additional to that undertaken for diagnostic radiology, is desirable. Specific additional training should be planned when new $x$ ray systems or techniques are implemented in a centre".

During the last years, much effort has been made to produce training material to help in the improvement of RP in cardiology laboratories. ${ }^{8-11}$ Cardiology scientific societies should promote training activities in RP to maintain a high level of radiation safety in the practice of interventional procedures.

\section{STAFF PROTECTION AND ITS RELATION TO PATIENT PROTECTION}

Staff RP cannot be treated independently from patient protection, as they correlate in many aspects. In general, staff lower their own level of risk if they are aware of RP for their patients. Radiation received by specialists is mainly the scattered radiation from patients. Thus, if patients receive less radiation, staff will also be exposed to less scattered radiation.

However, correlation between patient and staff radiological risks is not simple. As described in the article by Kuon and colleagues ${ }^{12}$ in this issue of Heart, many factors can influence occupational doses for the same radiation dose imparted to a patient. One of the most important factors is that protection tools are available in catheterisation laboratories and are used appropriately. Garments, lead goggles, caps (evaluated by Kuon and colleagues ${ }^{12}$ ), ceiling suspended shields, curtains under the table and other protective equipment provide a significant reduction in occupational

Abbreviations: ACC, American College of Cardiology; ICRP, International Commission on Radiological Protection; $R P$, radiological protection 
doses. The question is why these tools are not used in all catheterisation laboratories and in all procedures. One likely reason is the lack of information and training in RP. Thus, evaluation of these protective tools in routine clinical practice should occur in all laboratories.

Further, the use of protective tools may be sometimes self defeating for the patient. Protective resources such as gloves could lengthen the procedure in some cases and thus compromise the security and protection of the patient, as the tactile sensation of the catheter is reduced. In addition, the use of a leaded screen suspended from the ceiling could inhibit the movement of the C-arm $x$ ray system in some cases. In these situations, the cardiologist should have sufficient knowledge of the RP fundamentals to act in the most conscientious way for both doctor and patient.

New protective materials, lighter in weight than the older leaded clothes, require some attention in order to establish a new balance regarding protective features, acquisition costs, and spine lesion risk reduction because of lower weight.

In any case, the correlation between occupational and patient doses is very dependent on equipment, the specialist, and protocols followed throughout the procedure.

\section{PERSONAL DOSIMETRY}

It is necessary for interventional cardiologists to wear personal dosimeters on a regular basis, although this is not usually the standard practice. In some cases, dosimeters are not worn because of a lack of training in RP, which leads staff to disregard the dosimeters or the information that they provide, or to avoid problems with the regulatory authority in cases of exceeding the dose limits. ${ }^{13}$

Occupational dosimetry is critical for the personal safety of interventionists. The ICRP ${ }^{1}$ and the $\mathrm{ACC}^{5}$ recommend the use of two personal dosimeters, one worn outside the apron at the left shoulder or neck and the other worn under the apron at the waist. To monitor doses to the skin, hands, feet, and the lenses of eyes, special dosimeters (for example, a ring dosimeter) could be used for some special practices.

Personnel dosimetry monitors are typically worn for one month before being submitted for processing. The dosimeter placed outside the apron monitors exposure to the head, neck, and lenses of eyes, and it is important to ensure that the dose to the lens and thyroid are within recommended limits. Dose limits for occupational exposures are expressed in equivalent doses for deterministic effects in specific tissues and expressed in effective doses for stochastic effects throughout the body. See the glossary for details about limits, quantities, and units.

\section{Glossary of terms}

- Absorbed dose. A measure of the energy deposited in the unit of mass, measured in Gray (Gy).

- Dose limits. ${ }^{14}$ The limit on effective dose for exposed workers should be $100 \mathrm{mSv}$ in a consecutive five year period, subject to a maximum effective dose of $50 \mathrm{mSv}$ in any single year. The limit on equivalent dose for the lenses of the eye should be $150 \mathrm{mSv}$ in a year. The limit on equivalent dose for the skin should be $500 \mathrm{mSv}$ in a year. The limit on equivalent dose for the hands, forearms, feet, and ankles should be $500 \mathrm{mSv}$ in a year.

- Effective dose. Measures the global risk of the person exposed to ionising radiation and takes into account the equivalent doses in the different tissues and the radiosensitivity of that tissue. This quantity can be related with the increases of cancer and hereditary effects. It is also measured in Sieverts (Sv)

- Equivalent dose. Takes into account the kind of radiation imparting the energy, measured in Sieverts (Sv). For $x$ rays, it is numerically equal to the absorbed dose.
Doses in cardiology departments should be analysed and high doses and outliers should be investigated.

\section{PRACTICAL ADVICE TO IMPROVE STAFF RP}

- One of the most important radiation protection measures is to increase one's distance from the radiation source (the patient in the catheterisation laboratory). Working at $80 \mathrm{~cm}$ from the isocentre instead of $40 \mathrm{~cm}$ can decrease scattered dose to approximately a quarter of the original dose.

- Use a ceiling suspended screen and other structural or personal shielding tools available, such as a lead apron and thyroid collar, when possible.

- Minimise the use of fluoroscopy and use low fluoroscopy modes (for example, pulsed fluoroscopy) when possible.

- As scattered dose during cine is much higher than during fluoroscopy, acquire only the necessary number of frames per series and limit the number of series.

- As dose levels are more important for large patients and during very angulated projections, staff should be better protected in these cases.

- Scattered radiation is higher at the side of the $x$ ray tube and less important at the side of the image intensifier during lateral projections.

- Collimation of the radiation field (and generally all other factors reducing patient dose) decreases the level of scattered dose.

In addition, a final general recommendation: be aware of the RP of your patient and you will also be improving your own occupational protection.

\section{REFERENCES}

1 International Commission on Radiological Protection. ICRP Publication 85. Avoidance of radiation injuries from medical interventional procedures. Annals ICRP 2000;30(2). Oxford: Pergamon, Elsevier Science Ltd.

2 Vano E, Gonzalez L, Guibelalde E, et al. Radiation exposure to medical staff in interventional and cardiac radiology. Br J Radiol 1998:71:954-60.

3 Vano E, Gonzalez L, Beneytez F, et al. Lens injuries induced by occupational exposure to non-optimized interventional radiology laboratories. Br J Radiol 1998;71:728-33.

4 International Electrotechnical Commission. Medical electrical equipment. Part 2-43: particular requirements for the safety of $X$-ray equipment for interventional procedures. IEC 60601-2-43. 1 st ed. Geneva: International Electrotechnical Commission, 2000.

5 American College of Cardiology. Radiation safety in the practice of cardiology. ACC expert consensus document. J Am Coll Cardiol 1998:31:892-913.

6 European Commission. Guidelines on education and training in radiation protection for medical exposures. Radiation Protection 116. European Commission. Directorate General Environment, Nuclear Safety and Civil Protection. Luxembourg, 2000. URL: http://europa.eu.int/ comm/environment/radprot

7 Wilde P, Pitcher EM, Slack K. Radiation hazards for the patient in cardiological procedures. Heart 2001;85:127-30.

8 Wagner LK, Archer BR. Minimising risks from fluoroscopic $x$ rays, 3rd ed. The Woodlands, Texas: Partners in Radiation Management (RM Partnership), 2000

9 Balter S. Radiation safety in the cardiac catheterization laboratory: operational radiation safety. Catheter Cardiovasc Interv 1999;47:347-53.

10 Balter S. Interventional fluoroscopy. Physics, technology and safety. New York: Wiley-Liss, 2001

11 MARTIR, (Multimedia and Audiovisual Radiation Protection Training in Interventional Radiology). CD-ROM. Radiation Protection 119, European Commission. Directorate General Environment, Nuclear Safety and Civil Protection. Luxembourg, 2002 (free availability from the Publication Department of the European Commission, Office for Official Publications of the European Communities, Luxembourg: env-radprot@cec.eu.int).

12 Kuon E, Birkel J, Schmitt M, et al. Radiation exposure benefit of a lead cap in invasive cardiology. Heart 2003;89:1205-16.

13 Niklason LT, Marx MV, Chan HP. Interventional radiologists: occupational radiation doses and risks. Radiology 1993;187:729-33.

14 Council Directive, 96/29/Euratom of 13 May 1996 laying down basic safety standards for the protection of the health of workers and the general public against the dangers arising from ionizing radiation. Official journal No. L 159, 29 June 1996, 1-114. 